\title{
Advantages and Drawbacks of the ISM Method in Globular Cluster Photometry
}

\author{
József M. Benkő \\ Konkoly Observatory of the Hungarian Academy of Sciences, \\ P.O. Box 67. H-1525 Budapest, Hungary
}

\begin{abstract}
In the framework of our survey of variable stars in galactic globular clusters, we tested the promising new photometric technique, the Image Subtraction Method (Alard \& Lupton 1998, Alard 2000). We found that the standard deviations of light curves are much better compared to those of the more common PSF methods (e.g. DAOPHOT), especially in the most crowded regions. Moreover, many additional variable stars have become measurable with this method. Unlike previously published data we have transformed our results into conventional magnitudes. The accuracies of the derived magnitudes are discussed.
\end{abstract}

All previously published data where ISM photometry was applied to globular clusters (Olech et al. 1999, Kopacki 2000), were in relative units (e.g. $\left.e^{-} / \mathrm{ADU}\right)$ and this is not an accident! The big strength of ISM - that it is a relative method - is at the same time its most serious drawback. The method serves to determine differential fluxes for the variables relative to their fluxes in the reference frame, that is: $\Delta I_{i}^{(j)}=I_{0}^{(j)}-I_{i}^{(j)}$, where $I_{0}^{(j)}$ and $I_{i}^{(j)}$ are the fluxes of the $j$ th variable in the reference image and in the $i$ th frame, respectively. There are two possible ways to convert these into the usual instrumental magnitudes.

[1] If both magnitudes $\left(m_{0}^{(j)}\right)$ and fluxes $\left(I_{0}^{(j)}\right)$ for each variable can be measured in the reference frame, we simply apply the formula (see also Woźniak 2000)

$$
m_{i}^{(j)}=2.5 \log _{10} \frac{I_{0}^{(j)}}{I_{0}^{(j)}-\Delta I_{i}^{(j)}}+m_{0}^{(j)} .
$$

[2] If we know the magnitude values of two arbitrary points (e.g., $m_{1}^{(j)}=$ $\left.m_{\max }^{(j)}, m_{2}^{(j)}=m_{\min }^{(j)}\right)$ of the light curve, and we have $\Delta I_{1,2}$ values corresponding to the same phase, we can calculate the values $I_{0}$ and $m_{0}$. For each star

$$
\begin{aligned}
I_{0} & =\frac{\Delta I_{1} 10^{\left(m_{1}-m_{2}\right) / 2.5}-\Delta I_{2}}{10^{\left(m_{1}-m_{2}\right) / 2.5}-1}, \\
\text { and } \quad m_{0} & =m_{k}-2.5 \log _{10} \frac{I_{0}}{I_{0}-\Delta I_{k}}, \text { where } \mathrm{k}=1 \text { or } 2 .
\end{aligned}
$$

These transformations provide excellent results if the star is located in a relatively sparse region, where the traditional PSF methods provide accurate enough reference values $\left(m_{0}^{(j)}, I_{0}^{(j)}\right)$. In other cases the transformed light curves will be strongly distorted. To obtain reliable results the reference magnitudes have to be known within some hundredths of a magnitude. 

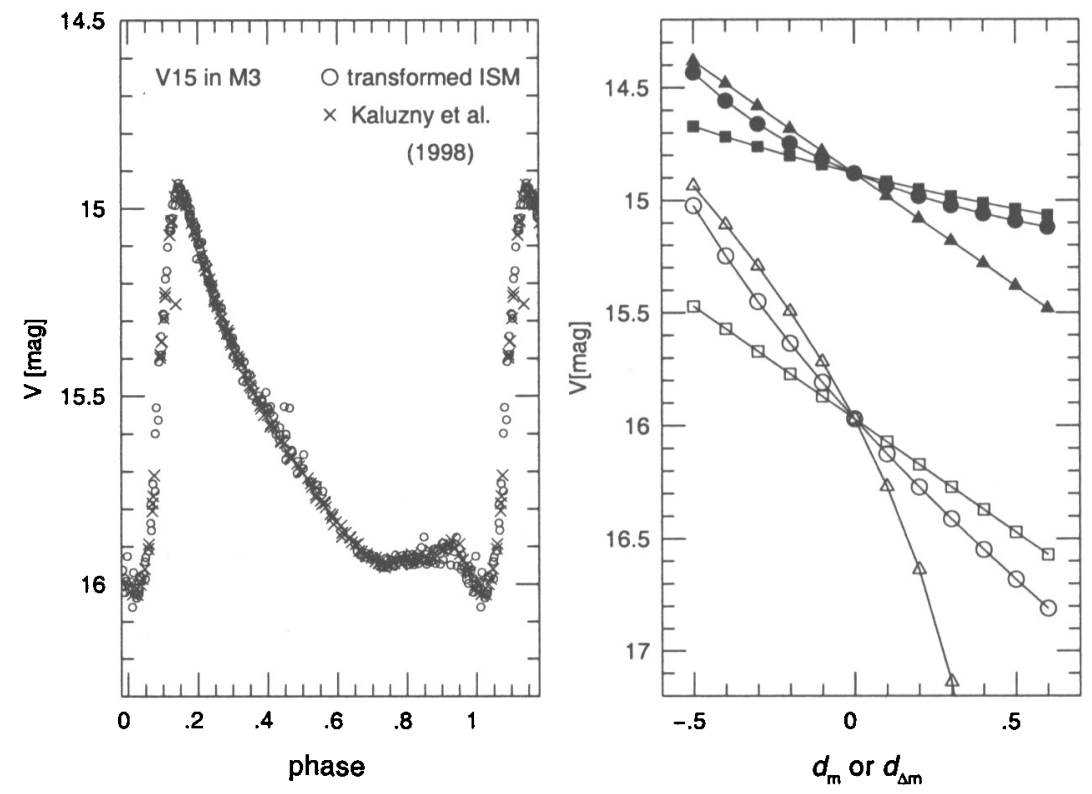

Figure 1. (left) An example for accurate magnitude transformation compared to previous DoPhot photometric results. (right) The effects of errors in magnitude zero points and amplitudes on the shape of the transformed light curve of an RR Lyrae star with $\left\langle m_{\mathrm{V}}\right\rangle=15.5$ and $1^{\mathrm{m}}$ total amplitude. $d_{m}$ and $d_{\Delta m}$ represent the errors of the reference magnitudes and amplitude, respectively. Empty and filled symbols respectively signal the transformed magnitudes of minima and maxima. Triangles and squares show the effect of $d_{m}$ errors in $m_{0}=m_{\max }$ and $m_{0}=m_{\min }$ respectively. If we know the total amplitude $\left(\Delta m=m_{\min }-\right.$ $\left.m_{\max }\right)$ with an error of $d_{\Delta m}$, the transformed light curve will be located between the lines denoted by circles.

Acknowledgments. I would like to thank J. Jurcsik for her continuous help. This work is partially supported by OTKA, Grants No. T-30954 and T-24022.

\section{References}

Alard C. 2000 A\&AS, 144, 363

Alard C. and Lupton R.H. 1998 ApJ, 503, 325

Kaluzny J., Hilditch R.W., Clement C., Rucinski S.M. 1998 MNRAS, 296, 347

Kopacki G. 2000 A\&A, 358, 547

Olech A., Woźniak P.R., Alard C., Kaluzny J., Thompson I.B. 1999 MNRAS, 310,759

Woźniak P.R., 2000 Acta Astron., 50, 421 